International Journal of Pure and Applied Mathematics

Volume 96 No. 3 2014, 363-376

ISSN: 1311-8080 (printed version); ISSN: 1314-3395 (on-line version)

url: http://www.ijpam.eu

doi: http://dx.doi.org/10.12732/ijpam.v96i3.7

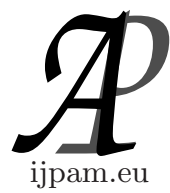

\title{
THE MODELING AND STABILITY PROBLEM FOR A COMMUNICATION CHANNEL SYSTEM WITH TRANSMITTER BREAKDOWN
}

\author{
Zvi Retchkiman Königsberg \\ Instituto Politecnico Nacional, CIC \\ Mineria 17-2, Col. Escandon, Mexico D.F. 11800, MEXICO
}

\begin{abstract}
In this work, the modeling and stability problem for a communication channel system with transmitter breakdown is considered. Timed Petri nets is the mathematical and graphical modeling technique utilized. Lyapunov stability theory provides the required tools needed to aboard the stability problem. Employing Lyapunov methods, a sufficient condition for stabilization is obtained. It is shown that it is possible to restrict the communication channel system with transmitter breakdown state space in such a way that boundedness is guaranteed. However, this restriction results to be vague. This inconvenience is overcome by considering a specific recurrence equation, in the max-plus algebra, which is assigned to the timed Petri net graphical model.
\end{abstract}

AMS Subject Classification: 08A99, 93D35, 93D99, 39A11, 08C99, 16Y60, 65F15, 05C50, 15A29, 15A33

Key Words: communication channel system, transmitter breakdown, discrete event dynamical systems, max-plus algebra, Lyapunov method, timed Petri nets

\section{Introduction}

A communication channel system can be considered as a dynamical system whose state evolves in time by the occurrence of events at possibly irregular time intervals, (therefore belong to the class of dynamical systems known as discrete event systems). Place-transitions Petri nets (commonly called Petri nets)

Received: July 12, 2014

(c) 2014 Academic Publications, Ltd. url: www.acadpubl.eu 
are a graphical and mathematical modeling tool that can be applied to the communication channel system in order to represent its states evolution. Petri nets are known to be useful for analyzing the systems properties in addition of being a paradigm for describing and studying information processing systems. Timed Petri nets are an extension of Petri nets, where now the timing at which the state changes is taken into consideration. This is of critical importance since it allows to consider useful measures of performance as for example: how long does the communication channel system spends at a given state etc. For a detailed discussion of Petri net theory see [1] and the references quoted therein. One of the most important performance issues to be considered in a communication channel system is its stability. Lyapunov stability theory provides the required tools needed to aboard the stability problem for communication channel systems modeled with timed Petri nets whose mathematical model is given in terms of difference equation. By proving practical stability one is allowed to preassigned the bound on the communication channel systems dynamics performance. Moreover, employing Lyapunov methods, a sufficient condition for the stabilization problem is also obtained. It is shown that it is possible to restrict the communication channel systems state space in such a way that boundedness is guaranteed. However, this restriction results to be vague. This inconvenience is overcome by considering a specific recurrence equation, in the max-plus algebra, which is assigned to the the timed Petri net graphical model. This paper is about the modeling and stability problem for communication channel systems with transmitter breakdown. The paper is organized as follows. In section 2 , Lyapunov theory for discrete event systems modeled with Petri nets is given. Section 3, presents max-plus algebra. In section 4, the solution to the stability problem for discrete event systems modeled with timed Petri nets is considered - Finally, in section 5 the modeling and stability analysis for communication channel systems with transmitter breakdown is addressed. Some conclusion remarks are also provided.

\section{Lyapunov Stability and Stabilization of Discrete Event Systems modeled with Petri Nets}

Notation. $N=\{0,1,2, \ldots\}, R_{+}=[0, \infty), N_{n_{0}}^{+}=\left\{n_{0}, n_{0}+1, \ldots, n_{0}+k, \ldots\right\}$, $n_{0} \geq 0$. Given $x, y \in R^{n}, x \leq y$ is equivalent to $x_{i} \leq y_{i}, \forall i$. A function $f(n, x)$, $f: N_{n_{0}}^{+} \times R^{n} \rightarrow R^{n}$ is called nondecreasing in $x$ if given $x, y \in R^{n}$ such that $x \geq y$ and $n \in N_{n_{0}}^{+}$then, $f(n, x) \geq f(n, y)$. 
Consider systems of first ordinary difference equations given by

$$
x(n+1)=f[n, x(n)], x\left(n_{o}\right)=x_{0}, n \in N_{n_{0}}^{+}
$$

where $n \in N_{n_{0}}^{+}, x(n) \in R^{n}$ and $f: N_{n_{0}}^{+} \times R^{n} \rightarrow R^{n}$ is continuous in $x(n)$.

Definition 1. The $n$ vector valued function $\Phi\left(n, n_{0}, x_{0}\right)$ is said to be a solution of (1) if $\Phi\left(n_{0}, n_{0}, x_{0}\right)=x_{0}$ and $\Phi\left(n+1, n_{0}, x_{0}\right)=f\left(n, \Phi\left(n, n_{0}, x_{0}\right)\right)$ for all $n \in N_{n_{0}}^{+}$.

Definition 2. The system (1) is said to be

i). Practically stable, if given $(\lambda, A)$ with $0<\lambda<A$, then

$$
\left|x_{0}\right|<\lambda \Rightarrow\left|x\left(n, n_{0}, x_{0}\right)\right|<A, \forall n \in N_{n_{0}}^{+}, n_{0} \geq 0 ;
$$

ii). Uniformly practically stable, if it is practically stable for every $n_{0} \geq 0$.

Definition 3. A continuous function $\alpha:[0, \infty) \rightarrow[0, \infty)$ is said to belong to class $\mathcal{K}$ if $\alpha(0)=0$ and it is strictly increasing.

Consider a vector Lyapunov function $v(n, x(n)), v: N_{n_{0}}^{+} \times R^{n} \rightarrow R_{+}^{p}$ and define the variation of $v$ relative to (1) by

$$
\Delta v=v(n+1, x(n+1))-v(n, x(n))
$$

Then, the following result concerns the practical stability of (1).

Theorem 4. Let $v: N_{n_{0}}^{+} \times R^{n} \rightarrow R_{+}^{p}$ be a continuous function in $x$, define the function $v_{0}(n, x(n))=\sum_{i=1}^{p} v_{i}(n, x(n))$ such that satisfies the estimates

$$
b(|x|) \leq v_{0}(n, x(n)) \leq a(|x|) ; a, b \in \mathcal{K}, \Delta v(n, x(n)) \leq w(n, v(n, x(n)))
$$

for $n \in N_{n_{0}}^{+}, x(n) \in R^{n}$, where $w: N_{n_{0}}^{+} \times R_{+}^{p} \rightarrow R^{p}$ is a continuous function in the second argument. Assume that : $g(n, e) \triangleq e+w(n, e)$ is nondecreasing in $e, 0<\lambda<A$ are given and finally that $a(\lambda)<b(A)$ is satisfied. Then, the practical stability properties of

$$
e(n+1)=g(n, e(n)), e\left(n_{0}\right)=e_{0} \geq 0 .
$$

imply the practical stability properties of system (1). 
Corollary 5. In Theorem (4):

i). If $w(n, e) \equiv 0$ we get uniform practical stability of (1) which implies structural stability.

ii). If $w(n, e)=-c(e)$, for $c \in \mathcal{K}$, we get uniform practical asymptotic stability of (1).

Definition 6. A Petri net is a 5-tuple, $P N=\left\{P, T, F, W, M_{0}\right\}$ where: $P=\left\{p_{1}, p_{2}, \ldots, p_{m}\right\}$ is a finite set of places, $T=\left\{t_{1}, t_{2}, \ldots, t_{n}\right\}$ is a finite set of transitions, $F \subset(P \times T) \cup(T \times P)$ is a set of arcs, $W: F \rightarrow N_{1}^{+}$is a weight function, $M_{0}: P \rightarrow N$ is the initial marking, $P \cap T=\varnothing$ and $P \cup T \neq \varnothing$.

Definition 7. The clock structure associated with a place $p_{i} \in P$ is a set $\mathbf{V}=\left\{V_{i}: p_{i} \in P\right\}$ of clock sequences $V_{i}=\left\{v_{i, 1}, v_{i, 2}, \ldots\right\}, v_{i, k} \in R^{+}, k=1,2, \ldots$

The positive number $v_{i, k}$, associated to $p_{i} \in P$, called holding time, represents the time that a token must spend in this place until its outputs enabled transitions $t_{i, 1}, t_{i, 2}, \ldots$, fire. We partition $P$ into subsets $P_{0}$ and $P_{h}$, where $P_{0}$ is the set of places with zero holding time, and $P_{h}$ is the set of places that have some holding time.

Definition 8. A timed Petri net is a 6-tuple $T P N=\left\{P, T, F, W, M_{0}, \mathbf{V}\right\}$ where $\left\{P, T, F, W, M_{0}\right\}$ are as before, and $\mathbf{V}=\left\{V_{i}: p_{i} \in P\right\}$ is a clock structure. A timed Petri net is a timed event petri net when every $p_{i} \in P$ has one input and one output transition, in which case the associated clock structure set of a place $p_{i} \in P$ reduces to one element $V_{i}=\left\{v_{i}\right\}$

Notice that if $W(p, t)=\alpha$ (or $W(t, p)=\beta$ ) then, this is often represented graphically by $\alpha,(\beta)$ arcs from $p$ to $t(t$ to $p)$ each with no numeric label.

Let $M_{k}\left(p_{i}\right)$ denote the marking (i.e., the number of tokens) at place $p_{i} \in P$ at time $k$ and let $M_{k}=\left[M_{k}\left(p_{1}\right), \ldots, M_{k}\left(p_{m}\right)\right]^{T}$ denote the marking (state) of $P N$ at time $k$. A transition $t_{j} \in T$ is said to be enabled at time $k$ if $M_{k}\left(p_{i}\right) \geq$ $W\left(p_{i}, t_{j}\right)$ for all $p_{i} \in P$ such that $\left(p_{i}, t_{j}\right) \in F$. It is assumed that at each time $k$ there exists at least one transition to fire. If a transition is enabled then, it can fire. If an enabled transition $t_{j} \in T$ fires at time $k$ then, the next marking for $p_{i} \in P$ is given by

$$
M_{k+1}\left(p_{i}\right)=M_{k}\left(p_{i}\right)+W\left(t_{j}, p_{i}\right)-W\left(p_{i}, t_{j}\right) .
$$

Let $A=\left[a_{i j}\right]$ denote an $n \times m$ matrix of integers (the incidence matrix) 
where $a_{i j}=a_{i j}^{+}-a_{i j}^{-}$with $a_{i j}^{+}=W\left(t_{i}, p_{j}\right)$ and $a_{i j}^{-}=W\left(p_{j}, t_{i}\right)$. Let $u_{k} \in\{0,1\}^{n}$ denote a firing vector where if $t_{j} \in T$ is fired then, its corresponding firing vector is $u_{k}=[0, \ldots, 0,1,0, \ldots, 0]^{T}$ with the one in the $j^{\text {th }}$ position in the vector and zeros everywhere else. The nonlinear difference matrix equation describing the dynamical behavior represented by a $P N$ is:

$$
M_{k+1}=M_{k}+A^{T} u_{k}
$$

where if at step $k, a_{i j}^{-}<M_{k}\left(p_{j}\right)$ for all $p_{i} \in P$ then, $t_{i} \in T$ is enabled and if this $t_{i} \in T$ fires then, its corresponding firing vector $u_{k}$ is utilized in the difference equation to generate the next step. Notice that if $M^{\prime}$ can be reached from some other marking $M$ and, if we fire some sequence of $d$ transitions with corresponding firing vectors $u_{0}, u_{1}, \ldots, u_{d-1}$ we obtain that

$$
M^{\prime}=M+A^{T} u, u=\sum_{k=0}^{d-1} u_{k} .
$$

Let $\left(N_{n_{0}}^{m}, d\right)$ be a metric space where $d: N_{n_{0}}^{m} \times N_{n_{0}}^{m} \rightarrow R_{+}$is defined by

$$
d\left(M_{1}, M_{2}\right)=\sum_{i=1}^{m} \zeta_{i}\left|M_{1}\left(p_{i}\right)-M_{2}\left(p_{i}\right)\right| ; \zeta_{i}>0
$$

and consider the matrix difference equation which describes the dynamical behavior of the discrete event system modeled by a $P N$, see (7).

Proposition 9. Let $P N$ be a Petri net. $P N$ is uniform practical stable if there exists a $\Phi$ strictly positive $m$ vector such that

$$
\Delta v=u^{T} A \Phi \leq 0
$$

Moreover, $P N$ is uniform practical asymptotic stable if the following equation holds

$$
\Delta v=u^{T} A \Phi \leq-c(e), c \in \mathcal{K}
$$

Lemma 10. Let suppose that Proposition (9) holds then,

$$
\Delta v=u^{T} A \Phi \leq 0 \Leftrightarrow A \Phi \leq 0
$$

Remark 11. Notice that since the state space of a TPN is contained in the state space of the same now not timed PN, stability of PN implies stability of the TPN. 


\subsection{Lyapunov Stabilization}

Definition 12. Let $P N$ be a Petri net. $P N$ is said to be stabilizable if there exists a firing transition sequence with transition count vector $u$ such that system (7) remains bounded.

Proposition 13. Let $P N$ be a Petri net. $P N$ is stabilizable if there exists a firing transition sequence with transition count vector $u$ such that the following equation holds

$$
\Delta v=A^{T} u \leq 0
$$

Remark 14. By fixing a particular $u$, which satisfies (11), the state space is restricted to those markings that are finite.

\section{Max-Plus Algebra}

\subsection{Basic Definitions}

Notation. $\epsilon=-\infty, e=0, \mathbb{R}_{\max }=\mathbb{R} \cup\{\epsilon\}, \underline{n}=1,2, \ldots, n$. Let $a, b \in \mathbb{R}_{\max }$ and define the operations $\oplus$ and $\otimes$ by: $a \oplus b=\max (a, b)$ and $a \otimes b=a+b$.

Definition 15. The set $\mathbb{R}_{\max }$ with the two operations $\oplus$ and $\otimes$ is called a max-plus algebra and is denoted by $\Re_{\max }=\left(\mathbb{R}_{\max }, \oplus, \otimes, \epsilon, e\right)$.

Definition 16. A semiring is a nonempty set $R$ endowed with two operations $\oplus_{R}, \otimes_{R}$, and two elements $\epsilon_{R}$ and $e_{R}$ such that: $\oplus_{R}$ is associative and commutative with zero element $\epsilon_{R}, \otimes_{R}$ is associative, distributes over $\oplus_{R}$, and has unit element $e_{R}, \epsilon_{R}$ is absorbing for $\otimes_{R}$ i.e., $a \otimes_{R} \epsilon=\epsilon_{R} \otimes a=a, \forall a \in R$.

In addition if $\otimes_{R}$ is commutative then $R$ is called a commutative semiring , and if $\oplus_{R}$ is such that $a \oplus_{R} a=a, \forall a \in R$ then it is called idempotent.

Theorem 17. The max-plus algebra $\Re_{\max }=\left(\mathbb{R}_{\max }, \oplus, \otimes, \epsilon, e\right)$ has the algebraic structure of a commutative and idempotent semiring. 


\subsection{Matrices and Graphs}

Let $\mathbb{R}_{\max }^{n \times n}$ be the set of $n \times n$ matrices with coefficients in $\mathbb{R}_{\max }$ with the following operations: The sum of matrices $A, B \in \mathbb{R}_{\max }^{n \times n}$, denoted $A \oplus B$ is defined by: $(A \oplus B)_{i j}=a_{i j} \oplus b_{i j}=\max \left(a_{i j}, b_{i j}\right)$ for $i$ and $j \in \underline{n}$. The product of matrices $A \in \mathbb{R}_{m a x}^{n \times l}, B \in \mathbb{R}_{\max }^{l \times n}$, denoted $A \otimes B$ is defined by: $(A \otimes B)_{i k}=$ $\bigotimes_{j=1}^{l}\left(a_{i j} \otimes b_{j k}\right)$ for $i$ and $k \in \underline{n}$.

Let $\mathcal{E} \in \mathbb{R}_{\max }^{n \times n}$ denote the matrix with all its elements equal to $\epsilon$ and denote by $E \in \mathbb{R}_{\max }^{n \times n}$ the matrix which has its diagonal elements equal to $e$ and all the other elements equal to $\epsilon$. Then, the following result can be stated.

Theorem 18. The 5-tuple $\Re_{\max }^{n \times n}=\left(\mathbb{R}_{\max }^{n \times n}, \oplus, \otimes, \mathcal{E}, E\right)$ has the algebraic structure of a noncommutative idempotent semiring.

Definition 19. Let $A \in \mathbb{R}_{\max }^{n \times n}$ and $k \in \mathbb{N}$ then the k-th power of $A$ denoted by $A^{\otimes k}$ is defined by: $A^{\otimes k}=A \otimes A \otimes \cdots \otimes A,(k$ times $)$, where $A^{\otimes 0}$ is set equal to $E$.

Definition 20. A matrix $A \in \mathbb{R}_{\max }^{n \times n}$ is said to be regular if $A$ contains at least one element distinct from $\epsilon$ in each row.

Definition 21. Let $\mathcal{N}$ be a finite and non-empty set and consider $\mathcal{D} \subseteq$ $\mathcal{N} \times \mathcal{N}$. The pair $G=(\mathcal{N}, \mathcal{D})$ is called a directed graph, where $\mathcal{N}$ is the set of elements called nodes and $\mathcal{D}$ is the set of ordered pairs of nodes called arcs. A directed graph $G=(\mathcal{N}, \mathcal{D})$ is called a weighted graph if a weight $w(i, j) \in \mathbb{R}$ is associated with any $\operatorname{arc}(i, j) \in \mathcal{D}$.

Let $A \in \mathbb{R}_{\max }^{n \times n}$ be any matrix, a graph $\mathcal{G}(A)$, called the communication graph of $A$, can be associated as follows. Define $\mathcal{N}(A)=\underline{n}$ and a pair $(i, j) \in \underline{n} \times \underline{n}$ will be a member of $\mathcal{D}(A) \Leftrightarrow a_{j i} \neq \epsilon$, where $\mathcal{D}(A)$ denotes the set of arcs of $\mathcal{G}(A)$.

Definition 22. A path from node $i$ to node $j$ is a sequence of $\operatorname{arcs} p=$ $\left\{\left(i_{k}, j_{k}\right) \in \mathcal{D}(A)\right\}_{k \in \underline{m}}$ such that $i=i_{1}, j_{k}=i_{k+1}$, for $k<m$ and $j_{m}=j$. The path $p$ consists of the nodes $i=i_{1}, i_{2}, \ldots, i_{m}, j_{m}=j$ with length $m$ denoted by $|p|_{1}=m$. In the case when $i=j$ the path is said to be a circuit. A circuit is said to be elementary if nodes $i_{k}$ and $i_{l}$ are different for $k \neq l$. A circuit 
consisting of one arc is called a self-loop.

Let us denote by $P(i, j ; m)$ the set of all paths from node $i$ to node $j$ of length $m \geq 1$ and for any arc $(i, j) \in \mathcal{D}(A)$ let its weight be given by $a_{i j}$ then the weight of a path $p \in P(i, j ; m)$ denoted by $|p|_{w}$ is defined to be the sum of the weights of all the arcs that belong to the path. The average weight of a path $p$ is given by $|p|_{w} /|p|_{1}$. Given two paths, as for example, $p=\left(\left(i_{1}, i_{2}\right),\left(i_{2}, i_{3}\right)\right)$ and $q=\left(\left(i_{3}, i_{4}\right),\left(\left(i_{4}, i_{5}\right)\right.\right.$ in $\mathcal{G}(A)$ the concatenation of paths $\circ: \mathcal{G}(A) \times \mathcal{G}(A) \rightarrow \mathcal{G}(A)$ is defined as $p \circ q=\left(\left(i_{1}, i_{2}\right),\left(i_{2}, i_{3}\right),\left(i_{3}, i_{4}\right),\left(i_{4}, i_{5}\right)\right)$.

The communication graph $\mathcal{G}(A)$ and powers of matrix $A$ are closely related as it is shown in the next theorem.

Theorem 23. Let $A \in \mathbb{R}_{\max }^{n \times n}$, then $\forall k \geq 1:\left[A^{\otimes k}\right]_{j i}=\max \left\{|p|_{w}: p \in\right.$ $P(i, j ; k)\}$, where $\left[A^{\otimes k}\right]_{j i}=\epsilon$ in the case when $P(i, j ; k)$ is empty i.e., no path of length $k$ from node $i$ to node $j$ exists in $\mathcal{G}(A)$.

Definition 24. Let $A \in \mathbb{R}_{\max }^{n \times n}$ then define the matrix $A^{+} \in \mathbb{R}_{\max }^{n \times n}$ as: $A^{+}=\bigoplus_{k=1}^{\infty} A^{\otimes k}$. Where the element $\left[A^{+}\right]_{j i}$ gives the maximal weight of any path from $j$ to $i$. If in addition one wants to add the possibility of staying at a node then one must include matrix $E$ in the definition of matrix $A^{+}$giving rise to its Kleene star representation defined by: $A^{*}=\bigoplus_{k=0}^{\infty} A^{\otimes k}$.

Lemma 25. Let $A \in \mathbb{R}_{\max }^{n \times n}$ be such that any circuit in $\mathcal{G}(A)$ has average circuit weight less than or equal to $\epsilon$. Then it holds that: $A^{*}=\bigoplus_{k=0}^{n-1} A^{\otimes k}$.

Definition 26. Let $G=(\mathcal{N}, \mathcal{D})$ be a graph and $i, j \in \mathcal{N}$, node $j$ is reachable from node $i$, denoted as $i \mathcal{R} j$, if there exists a path from $i$ to $j$. A graph $G$ is said to be strongly connected if $\forall i, j \in \mathcal{N}, j \mathcal{R} i$. A matrix $A \in \mathbb{R}_{\max }^{n \times n}$ is called irreducible if its communication graph is strongly connected, when this is not the case matrix $A$ is called reducible.

Definition 27. Let $G=(\mathcal{N}, \mathcal{D})$ be a not strongly connected graph and $i, j \in \mathcal{N}$, node $j$ communicates with node $i$, denoted as $i \mathcal{C} j$, if either $i=j$ or $i \mathcal{R} j$ and $j \mathcal{R} i$.

The relation $i \mathcal{C} j$ defines an equivalence relation in the set of nodes, and 
therefore a partition of $\mathcal{N}$ into a disjoint union of subsets, the equivalence classes, $\mathcal{N}_{1}, \mathcal{N}_{2}, \ldots, \mathcal{N}_{q}$ such that $\mathcal{N}=\mathcal{N}_{1} \cup \mathcal{N}_{2} \cup \ldots \cup \mathcal{N}_{q}$ or $\mathcal{N}=\bigcup_{i \in \mathcal{N}}[i] ;[i]=$ $\{j \in \mathcal{N}: i C j\}$.

Given the above partition, it is possible to focus on subgraphs of $G$ denoted by $G_{r}=\left(\mathcal{N}_{r}, \mathcal{D}_{r}\right) ; r \in \underline{q}$ where $\mathcal{D}_{r}$ denotes the subset of arcs, which belong to $\mathcal{D}$, that have both the begin node and end node in $\mathcal{N}_{r}$. If $\mathcal{D}_{r} \neq \varnothing$, the subgraph $G_{r}=\left(\mathcal{N}_{r}, \mathcal{D}_{r}\right)$ is known as a maximal strongly connected subgraph of $G$.

Definition 28. The reduced graph $\widetilde{G}=(\widetilde{\mathcal{N}}, \widetilde{\mathcal{D}})$ of $G$ is defined by setting $\widetilde{\mathcal{N}}=\left\{\left[i_{1}\right],\left[i_{2}\right], \ldots\left[i_{q}\right]\right\}$ and $\left(\left[i_{r}\right],\left[i_{s}\right]\right) \in \widetilde{\mathcal{D}}$ if $r \neq s$ and there exists an arc $(k, l) \in \mathcal{D}$ for some $k \in\left[i_{r}\right]$ and $l \in\left[i_{s}\right]$.

let $A_{r r}$ denote the matrix by restricting $A$ to the nodes in $\left[i_{r}\right] \forall r \in \underline{q}$ i.e., $\left[A_{r r}\right]_{k l}=a_{k l} \forall k, l \in\left[i_{r}\right]$. Then $\forall r \in \underline{q}$ either $A_{r r}$ is irreducible or is equal to $\epsilon$. Therefore since by construction the reduced graph does not contain any circuits, the original reducible matrix $A$ after a possible relabeling of the nodes in $G(A)$, can be written as:

$$
A=\left(\begin{array}{ccccc}
A_{11} & A_{12} & \cdots & \cdots & A_{1 q} \\
\mathcal{E} & A_{22} & \cdots & \cdots & A_{2 q} \\
\mathcal{E} & \mathcal{E} & A_{33} & & \vdots \\
\vdots & \vdots & \ddots & \ddots & \vdots \\
\mathcal{E} & \mathcal{E} & \cdots & \mathcal{E} & A_{q q}
\end{array}\right)
$$

with matrices $A_{s r} 1 \leq s<r \leq q$, where each finite entry in $A_{s r}$ corresponds to an arc from a node in $\left[i_{r}\right]$ to a node in $\left[i_{s}\right]$.

Definition 29. Let $A \in \mathbb{R}_{\max }^{n \times n}$ be a reducible matrix then, the block upper triangular given by (12) is said to be a normal form of matrix $A$.

\subsubsection{Spectral Theory and Linear Equations}

Definition 30. Let $A \in \mathbb{R}_{\max }^{n \times n}$ be a matrix. If $\mu \in R_{\max }$ is a scalar and $v \in R_{\max }^{n}$ is a vector that contains at least one finite element such that: $A \otimes v=\mu \otimes v$ then, $\mu$ is called an eigenvalue and $v$ an eigenvector.

Let $\mathcal{C}(A)$ denote the set of all elementary circuits in $\mathcal{G}(A)$ and write: $\lambda=$ $\max _{p \in \mathcal{C}(A)} \frac{|p|_{\mathrm{w}}}{|p|_{1}}$ for the maximal average circuit weight. Notice that since $\mathcal{C}(A)$ is a 
finite set, the maximum is attained (which is always the case when matrix $A$ is irreducible). In case $\mathcal{C}(A)=\emptyset$ define $\lambda=\epsilon$.

Definition 31. A circuit $p \in G(A)$ is said to be critical if its average weight is maximal. The critical graph of $A$, denoted by $G^{c}(A)=\left(\mathcal{N}^{c}(A), \mathcal{D}^{c}(A)\right)$, is the graph consisting of those nodes and arcs that belong to critical circuits in $G(A)$.

Theorem 32. If $A \in \mathbb{R}_{\max }^{n \times n}$ is irreducible, then there exists one and only one finite eigenvalue (with possible several eigenvectors). This eigenvalue is equal to the maximal average weight of circuits in $G(A) \lambda(A)=\max _{p \in \mathcal{C}(A)} \frac{|p|_{\mathrm{w}}}{|p|_{1}}$

Theorem 33. Let $A \in \mathbb{R}_{\max }^{n \times n}$ and $b \in \mathbb{R}_{\max }^{n}$. If the communication graph $G(A)$ has maximal average circuit weight less than or equal to $e$, then $x=A^{*} \otimes b$ solves the equation $x=(A \otimes x) \oplus b$. Moreover, if the circuit weights in $G(a)$ are negative then, the solution is unique.

\subsection{Max-Plus recurrence equations for timed event Petri Nets}

Definition 34. Let $A_{m} \in \mathbb{R}_{\max }^{n \times n}$ for $0 \leq m \leq M$ and $x(m) \in \mathbb{R}_{\max }^{n}$ for $-M \leq m \leq-1 ; M \geq 0$. Then, the recurrence equation: $x(k)=\bigoplus_{m=0}^{M} A_{m} \otimes$ $x(k-m) ; k \geq 0$ is called an $M$ th order recurrence equation.

Theorem 35. The $M$ th order recurrence equation, given by equation $x(k)=\bigoplus_{m=0}^{M} A_{m} \otimes x(k-m) ; k \geq 0$, can be transformed into a first order recurrence equation $x(k+1)=A \otimes x(k) ; k \geq 0$ provided that $A_{0}$ has circuit weights less than or equal to zero.

With any timed event Petri net, matrices $A_{0}, A_{1}, \ldots, A_{M} \in \mathbb{N}^{n} \times \mathbb{N}^{n}$ can be defined by setting $\left[A_{m}\right]_{j l}=a_{j l}$, where $a_{j l}$ is the largest of the holding times with respect to all places between transitions $t_{l}$ and $t_{j}$ with $m$ tokens, for $m=0,1, \ldots, M$, with $M$ equal to the maximum number of tokens with respect to all places. Let $x_{i}(k)$ denote the $k$ th time that transition $t_{i}$ fires, then the vector $x(k)=\left(x_{1}(k), x_{2}(k), \ldots x_{m}(k)\right)^{T}$, called the state of the system, satisfies the $M$ th order recurrence equation: $x(k)=\bigoplus_{m=0}^{M} A_{m} \otimes x(k-m) ; k \geq 0$ 
Now, assuming that all the hypothesis of theorem (35) are satisfied, and setting $\hat{x}(k)=\left(x^{T}(k), x^{T}(k-1), \ldots, x^{T}(k-M+1)\right)^{T}$, equation $x(k)=\bigoplus_{m=0}^{M} A_{m} \otimes$ $x(k-m) ; k \geq 0$ can be expressed as: $\hat{x}(k+1)=\hat{A} \otimes \hat{x}(k) ; k \geq 0$, which is known as the standard autonomous equation.

\section{The Solution to the stability Problem for Discrete Event Dynamical Systems Modeled with timed Petri Nets}

Definition 36. A TPN is said to be stable if all the transitions fire with the same proportion i.e., if there exists $q \in \mathbb{N}$ such that

$$
\lim _{k \rightarrow \infty} \frac{x_{i}(k)}{k}=q, \forall i=1, \ldots, n
$$

This means that in order to obtain a stable $T P N$ all the transitions have to be fired $q$ times. It will be desirable to be more precise and know exactly how many times. The answer to this question is given next.

Lemma 37. Consider the recurrence relation $x(k+1)=A \otimes x(k), k \geq 0$, $x(0)=x_{0} \in \mathbb{R}^{n}$ arbitrary. $A$ an irreducible matrix and $\lambda \in \mathbb{R}$ its eigenvalue then,

$$
\lim _{k \rightarrow \infty} \frac{x_{i}(k)}{k}=\lambda, \forall i=1, \ldots, n
$$

Proof. Let $v$ be an eigenvector of $A$ such that $x_{0}=v$ then,

$$
x(k)=\lambda^{\otimes k} \otimes v \Rightarrow x(k)=k \lambda+v \Rightarrow \frac{x(k)}{k}=\lambda+\frac{v}{k} \Rightarrow \lim _{k \rightarrow \infty} \frac{x_{i}(k)}{k}=\lambda
$$

Now starting with an unstable $T P N$, collecting the results given by: proposition (13), what has just been discussed about recurrence equations for $T P N$ at the end of subsection (3.3) and the previous lemma (37) plus theorem (32), the solution to the problem is obtained. 


\section{Modeling and Stability Analysis of Communication Channel Systems with Transmitter breakdown}

Consider a communication channel system with transmitter breakdown whose TPN model is depicted in Figure 1.

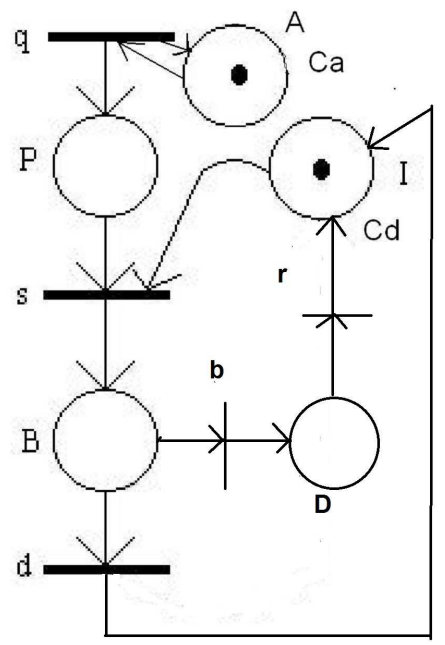

Figure 1. Transmitter breakdown timed Petri net model

Where the events (transitions) that drive the system are: q: receivers connect to the communication channel, s: messages are sent, b: the transmitter breaks, r: the transmission is restored, $d$ : the message has been successfully received. The places (that represent the states of the queue) are: A: set of receivers, $\mathrm{P}$ : the receiver is waiting for a message to be sent, B: the message is being received, D: transmitter out of order, I: the transmitter is idle. The holding times associated to the places $\mathrm{A}$ and $\mathrm{I}$ are $C a$ and $C d$ respectively, (with $C a>C d$ ). The incidence matrix that represents the $P N$ model is

$$
A=\left[\begin{array}{ccccc}
0 & 1 & 0 & 0 & 0 \\
0 & -1 & 1 & -1 & 0 \\
0 & 0 & -1 & 1 & 0 \\
0 & 0 & -1 & 0 & 1 \\
0 & 0 & 0 & 1 & -1
\end{array}\right]
$$

Therefore since there does not exists a $\Phi$ strictly positive $m$ vector such that $A \Phi \leq 0$ the sufficient condition for stability is not satisfied, (moreover, the $P N$ $(T P N)$ is unbounded since by the repeated firing of $\mathrm{q}$, the marking in $\mathrm{P}$ grows 
indefinitely). However, by taking $u=[k, k, k / 2, k / 2, k / 2] ; k>0$ (but unknown) we get that $A^{T} u \leq 0$. Therefore, the $P N$ is stabilizable which implies that the $T P N$ is stable. Now, let us proceed to determine the exact value of $k$. From the TPN model we obtain that:

$$
A_{0}=\left(\begin{array}{lllll}
\varepsilon & \varepsilon & \varepsilon & \varepsilon & \varepsilon \\
0 & \varepsilon & \varepsilon & \varepsilon & \varepsilon \\
\varepsilon & 0 & \varepsilon & \varepsilon & \varepsilon \\
\varepsilon & \varepsilon & \varepsilon & \varepsilon & \varepsilon \\
\varepsilon & \varepsilon & \varepsilon & 0 & \varepsilon
\end{array}\right)
$$

and

$$
A_{1}=\left(\begin{array}{ccccc}
C a & \varepsilon & \varepsilon & \varepsilon & \varepsilon \\
\varepsilon & \varepsilon & C d & \varepsilon & C d \\
\varepsilon & \varepsilon & \varepsilon & \varepsilon & \varepsilon \\
\varepsilon & \varepsilon & \varepsilon & \varepsilon & \varepsilon \\
\varepsilon & \varepsilon & \varepsilon & \varepsilon & \varepsilon
\end{array}\right)
$$

which implies

$$
A_{0}^{*}=\left(\begin{array}{ccccc}
0 & \varepsilon & \varepsilon & \varepsilon & \varepsilon \\
0 & 0 & \varepsilon & \varepsilon & \varepsilon \\
0 & 0 & 0 & \varepsilon & \varepsilon \\
\varepsilon & \varepsilon & \varepsilon & 0 & \varepsilon \\
\varepsilon & \varepsilon & \varepsilon & 0 & 0
\end{array}\right)
$$

leading to:

$$
\hat{A}=A_{0}^{*} \otimes A_{1}=\left(\begin{array}{ccccc}
C a & \varepsilon & \varepsilon & \varepsilon & \varepsilon \\
C a & \varepsilon & C d & \varepsilon & C d \\
C a & \varepsilon & C d & \varepsilon & C d \\
\varepsilon & \varepsilon & \varepsilon & \varepsilon & \varepsilon \\
\varepsilon & \varepsilon & \varepsilon & \varepsilon & \varepsilon
\end{array}\right)
$$

Therefore, $\lambda(A)=\max _{p \in \mathcal{C}(A)} \frac{|p|_{\mathrm{w}}}{|p|_{1}}=\max \{C a, C d\}=C a$. This means that in order for the TPN to be stable and work properly the speed at which the service operates has to be equal to $C a$ (the firing speed of transition q) which is attained by taking $k=C a$.

\section{Conclusions}

This paper studies the modeling and stability problem for communication channel systems with transmitter breakdown using timed Petri nets, Lyapunov 
methods and max-plus algebra. The results obtained are consistent to what was expected.

\section{References}

[1] T. Murata, Petri nets: Properties, analysis, and applications, Proc. IEEE, 77, No. 4, (1989).

[2] Z. Retchkiman, Stability theory for a class of dynamical systems modeled with Petri nets, International Journal of Hybrid Systems, 4, No. 1,(2005).

[3] Z. Retchkiman, From Stability to the Stabilization problem of Discrete event Systems modeled by Petri Nets, American Control Conference. (1999).

[4] B. Heidergott, G. J. Olsder, J. van der Woude, Max Plus at Work, Princeton University Press, (2006).

[5] F. Baccelli, G. Cohen, G. J. Olsder, J. P. Quadrat, Synchronization and Linearity, Web-edition, (2001). 